UCRL-ID-126257

\title{
Nondestructive Computed Tomography for Pit Inspections
}

\author{
H. Martz \\ C. Logan \\ J. Haskins \\ E. Johansson \\ D. Perkins \\ J. M. Hernandez \\ D. Schneberk \\ K. Dolan
}

February 7, 1997

This is an informal report intended primarily for internal or limited external diatribution. The opinions and conclusions etated are those of the author and may or may not be thoce of the Laboration. 


\section{DISCLAIMER}

This document was prepared as an account of work sponsored by an agency of the United States Government. Neither the United States Government mor the University of California nor any of their employees, makes any warranty, express or Implled, or assumes any legal liability or responsibility for the accuracy, completeness, or usefulness of any informatlon, apparatus, product, or process disclosed, or represents that its use would not Infringe privately owned rights. Reference herein to any specific commercial products, process, or service by trade name, trademark, manufacturer, or otherwise, does not necessarily constitute or imply its endorsement, recommendation, or favoring by the United States Government or the University of Callformis. The views and opinions of authors expressed herein do not necessarily state or reflect those of the United States Government or the University of Californis, and shall not be used for advertising or product endorsement purposes.

This report has been reproduced directly from the best avallable copy.

Avallable to DOE and DOE contractors from the Ofilice of Scientinc and Technical Information

P.O. Box 62, Oak Ridge, TN 37831

Prices available from (615) 576-8401, FTS 626-8401

Avallable to the public from the

National Technical Information Service

U.S. Department of Commerce

5285 Port Royal Rd.,

Springiteld, VA 22161 


\title{
Nondestructive Computed Tomography for Pit Inspections ${ }^{\dagger}$
}

\author{
Harry Martz, Clint Logan, Jerry Haskins, Erik Johansson, \\ Dwight Perkins, José M. Hernández, Dan Schneberk, and Ken Dolan \\ Lawrence Livermore National Laboratory, \\ Livermore, CA 94550
}

\section{Project Objectives:}

The overall objective of this project is to develop new approaches to electronically capture digital radiography (DR) and computed tomography (CT) images at high x-ray energies to satisfy spatial and contrast requirements for inspection of high-density weapons components.

Recent advances in the performance of camera-scintillator radiography systems have enabled new horizons in high-fidelity, high-energy imaging. In spite of these advances, some inspection requirements for weapons (e.g., 10-20 line-pair/mm or 50-25 $\mu \mathrm{m}$ spatial resolution at 4-9 MV in three dimensions) still exceed current capabilities. However, this fidelity is not necessarily needed for the entire weapon. Region of interest (ROI) imaging, the ability to image a portion of an object, holds the promise of delivering the required spatial resolution with currently available components. We are combining the highest performance camera-scintillator configurations with 4-9 MV x-ray sources and the best ROI algorithms for imaging key areas in weapons components.

\section{DR/CT Detector R\&D}

We have three ongoing DR/CT detector activities: (1) simulations, (2) design and construction, and (3) validation.

\section{Simulations}

We know from prior experience that radiation interactions in the camera and associated electronics degrade image quality. Since none of these components are exposed to the primary beam, these effects must arise from scattered radiation. All scattered radiation originates from something exposed to primary radiation. We decided to address in a quantitative manner the effects of geometry and materials of construction on scattered radiation.

We applied the TART Monte Carlo code to this task.[TART95] Our best estimate of the photon spectrum from our $9 \mathrm{MV}$ linac with a tungsten converter is a TIGER calculation done by Glenn Tersell.[TER92] The results are shown in the Figure 1.

\footnotetext{
† This work was funded by LDRD and performed under the auspices of the U.S. Department of Energy by the Lawrence Livermore National Laboratory under contract W-7405-ENG-48.
} 


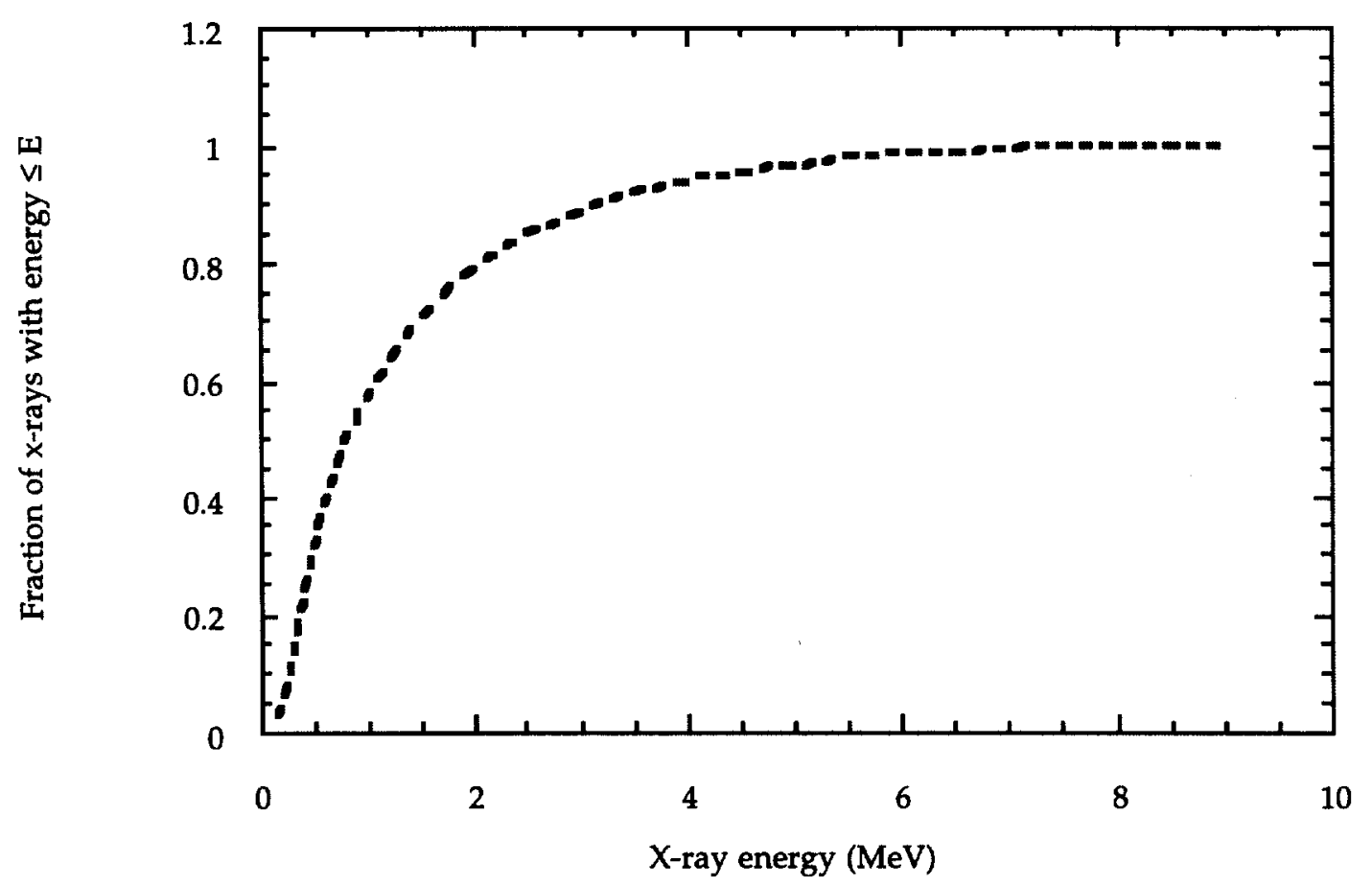

Figure 1 Calculated $\mathrm{x}$-ray spectrum from our $9 \mathrm{MV}$ electron linac with tungsten converter. One half the $\mathrm{x}$-rays have an energy less than $800 \mathrm{keV}$.

We used the spectrum of Figure 1 as the source spectrum for a set of TART calculations. We zoned the problem so that the source was directed down an axis incident on a small pellet of scattering mass (See Figure 2). The scattering pellet was surrounded by a spherical "detector" of $\mathrm{Si}$, which in turn was subdivided into zones by angle. We chose to make the detector of Si since experimentally we observe a noticeable effect that seems to arise from direct radiation hits on the CCD chip. This effect is hot or near-saturated detector elements.

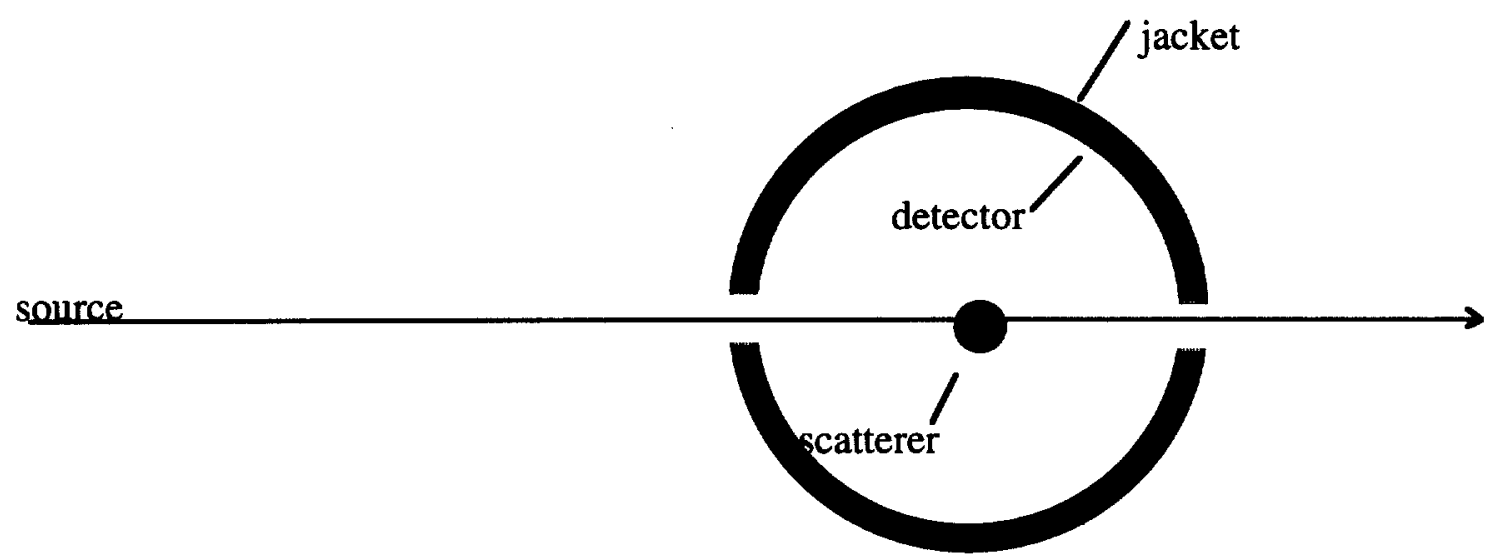

Figure 2 Schematic of geometry used for TART calculations. 
Our initial set of simulation problems also included the variable of the presence or absence of a spherical $\mathrm{Pb}$ jacket around the outside of the spherical Si detector. Our reasoning was that we wished to observe the effect of radiation return or scattering back from the jacket into the detector. Our analysis resulted in something quite different than we initially expected.

A schematic of the zoning for the TART calculation is shown in Figure 2. We sampled the effect of different scattering pellets by running $\mathrm{Be}, \mathrm{C}, \mathrm{Al}, \mathrm{Fe}, \mathrm{Pb}$, and $\mathrm{U}$. We kept the pellet constant in size and mass, changing only the element. Initially, we put air in the problem every place not otherwise occupied.

Examination of the initial runs revealed that the configuration with air in the jacket has greater energy deposition within the $\mathrm{Si}$ detector than when the jacket is $\mathrm{Pb}$. Additional calculations identified this effect as coming from air scatter X-rays into the Si detector from outside of the jacket. With the benefit of hindsight this result seems reasonable given that the mass of air presented to the primary beam exceeds the mass of the scattering pellet. With additional runs, we were also able to show that the air inside the detector sphere is a significant scatter source.

The results from the series of runs with a $\mathrm{Pb}$ jacket are useful to gain an understanding of the effects of material of construction and DR/CT imager design. These are summarized in Figure 3. The values given in Figure $3 d o$ include the contribution from air scatter within the spherical detector. The air inside the detector accounts for between 15 and $20 \%$ of the energy deposited in the detector and is the same for all cases shown.

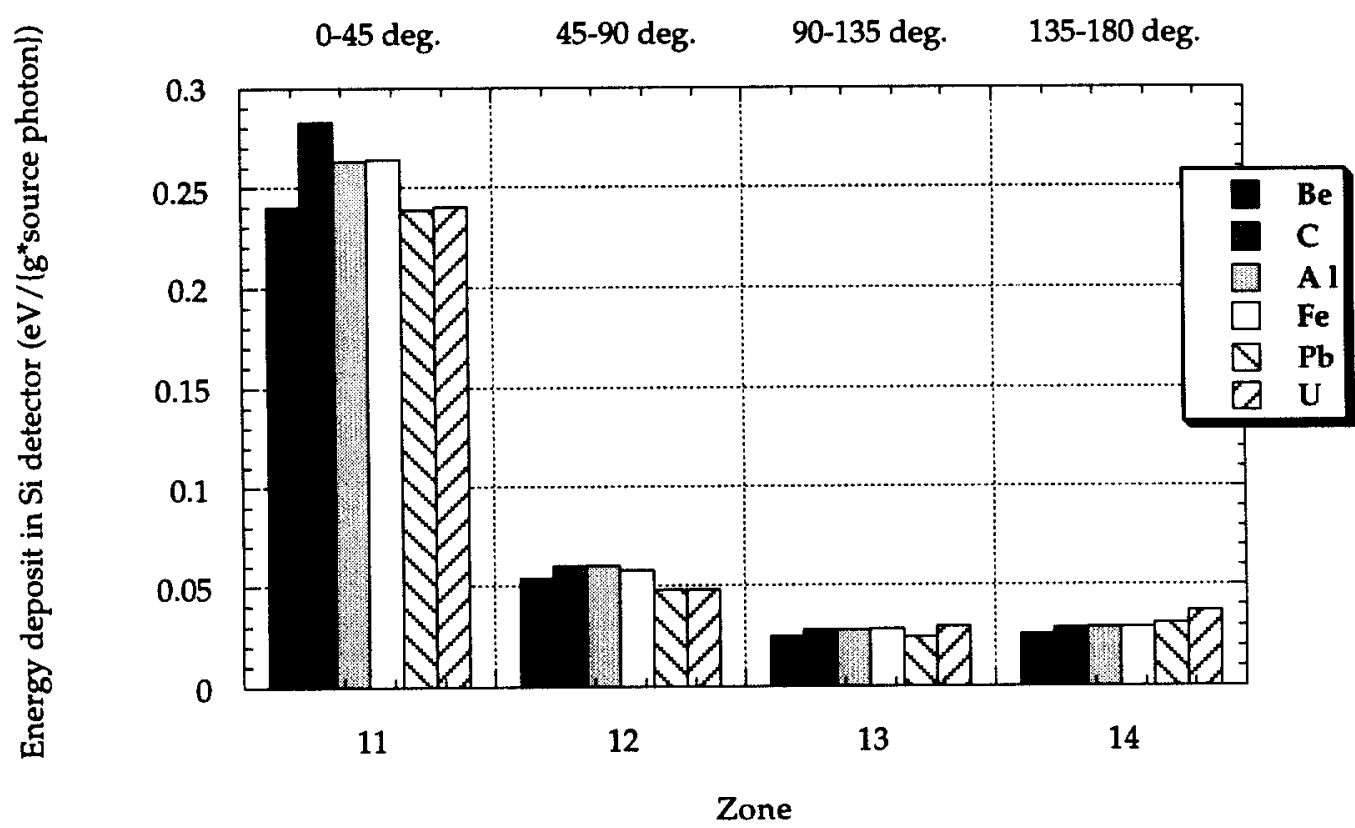

Figure 3 Energy deposited in the Si detector as a function of angle (zone) and scatterer composition. The energy deposit is expressed in units of eV into $\mathrm{Si} /$ (source photon)*(gram of scatter material). 
Three design rules emerge from these simulation results:

- Scatter is strongly forward peaked.

- It matters little what material is chosen for mirrors, mounts, light enclosures, scintillator frames etc., so long as they are low in mass.

- Scatter is directly proportional to mass within the primary beam.

- Air is a significant source of scattered x-rays.

We also have studied the effects of the scintillator on the imager design. Experiments have determined that focusing into a piece of scintillating glass has certain advantages. Los Alamos National Laboratory (LANL) researchers have conducted experiments to determine the optimum focus for various glass and camera parameters.[FRY96]

We have created a model that duplicates these results and allows us to predict optimum configuration of lens, glass thickness, and focus for an application given the $\mathrm{x}$-ray source energy spectrum and spatial resolution desired.[PER96] The conclusions reached from these simulations are, scintillator thickness, $\mathrm{x}$-ray energy and coatings all influence the location of the optimum focus. The best resolution is achieved:

- with an antireflection coating on both surfaces of the scintillator and a light absorbing material on the side of the glass toward the $\mathrm{x}$-ray source;

- by shortening the optical path and stopping down the lens, thus increasing depth of field while maintaining light gathering ability.

The lens size can then be chosen to achieve the desired field-of-view. We also found that a finite-conjugate lens provides higher spatial resolution than an infinite-conjugate lens in this application.

To date our modeling has centered on finding the optimal focus with respect to scintillating glass thickness and $\mathrm{x}$-ray source energy. We used the experiments performed at LANL by Fry et al.[FRY96] to validate our model. Figure 4 shows the effect of scintillating glass thickness and a mirror coating on the optimum focus at $100 \mathrm{keV}$. In this figure the depth of the optimum focus is from the camera side of the scintillator. The results agree well with the LANL experimental results, e.g., we found that the optimum focus for the 12-mm glass is $\sim 7.5 \mathrm{~mm}$; the LANL results were also $\sim 7.5 \mathrm{~mm}$.

For the cases shown in Figure 4, a reflective coating decreases the modulation transfer function (MTF) because the depth of field of the lens is insufficient to maintain sharp focus for all of the light. Future work will compare the relative MTF of various combinations of lens, scintillator and $\mathrm{x}$-ray source. 


\section{Focus vs Glass Thickness for $100 \mathrm{keV}$}

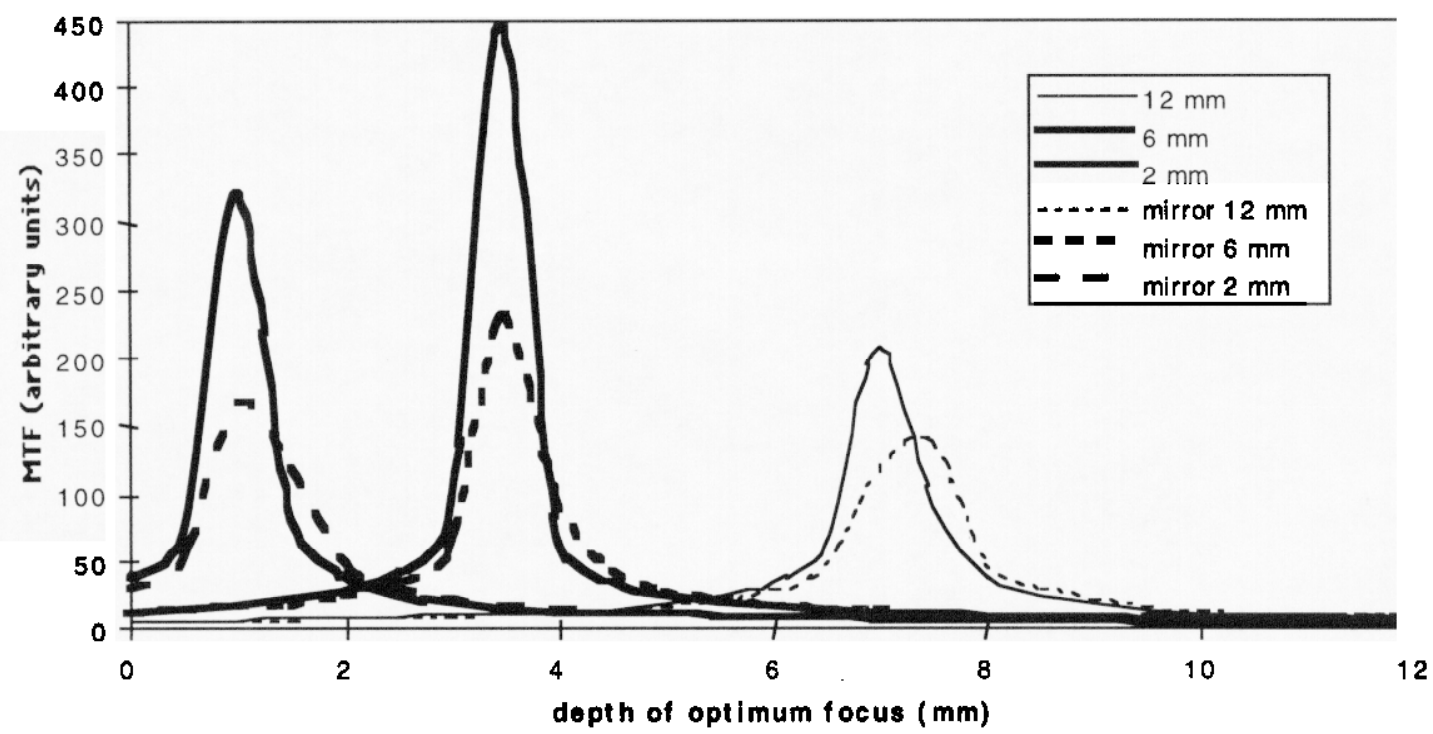

Figure 4 Location of the optimum focus as a function of scintillating glass thickness and presence of a mirror coating on the $\mathrm{x}$-ray side of the glass.

\section{Dynamic range required}

Weapons components of interest require a large detector dynamic range. We have applied 12-, 14- and 16-bit cameras to image $\mathrm{x}$-ray data converted into visible light by glass scintillator monolithic and fiber optic plates. In all cases we demonstrated improvements in dynamic range and contrast compared to 8-bit camera data. This data was obtained at medium $(200 \mathrm{kV})$ energy. Results for 14- and 8-bit cameras using a monolithic glass scintillator with mirror and anti-reflective coatings are shown in Figure 5.

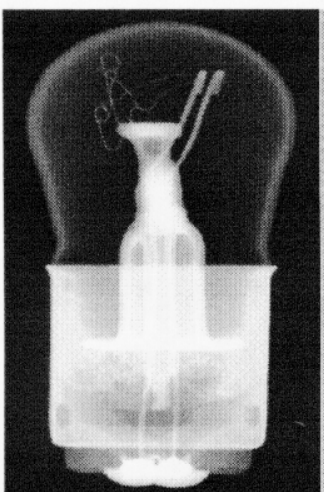

14 bit

Comparison of one 14 bit digital ima

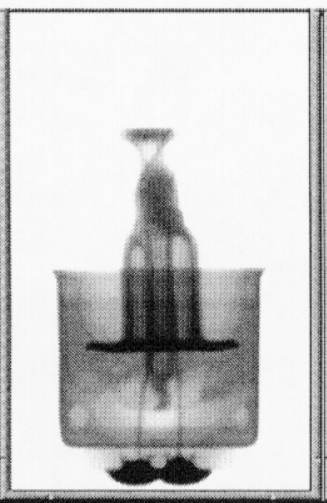

8 bit

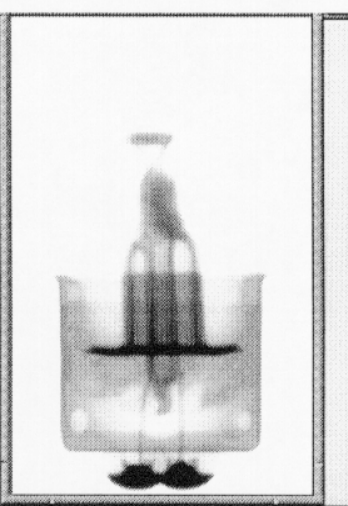

8 bit

作 multiple 8 bit eposures.

Figure 5. Digital radiographic images of a light bulb at $200 \mathrm{kV}$. 


\section{System description}

Based on these rules and our experience, we decided on building a camera system with two bending mirrors. This permits locating the CCD camera outside of a $45^{\circ}$ forward cone from the object under examination. This is especially important since the object is the largest mass item in the primary beam. The camera will have local shielding resembling a box with one end of the box made of leaded glass for the optical path. Since the leaded glass is the least effective portion of the shielding, it will be arranged to minimize the probability of a single scatter resulting in an $\mathrm{x}$-ray hitting the leaded glass.

Some portions of the light tight box enclosing the camera system will be illuminated by the primary beam. Since this enclosure serves only as a light barrier, it was constructed of aluminized mylar placed over an aluminum space frame to reduce the amount of mass around the detector. The inside of the mylar was covered with black cloth to reduce internal light reflections.

Design rules gained from Monte Carlo calculations were coupled with previous experience in scintillator glass CT systems to develop a new system for use with the 4 and $9 \mathrm{MV}$ linear accelerators. There are several unique features to this new system:

- two bending mirrors allow placement of the CCD camera within a low scatter area (i.e., $90-135^{\circ}$ );

- the position of the camera and scintillator are fixed with respect to each other;

- the mirrors are aligned with respect to the camera and scintillator and with respect to each other;

- the optical path length is variable to accommodate focusing and field-of-view changes.

The completed system is depicted in Figure 6. It allows fields-of-view ranging from approximately $5.0 \mathrm{~cm} \times 3.5 \mathrm{~cm}$ to $28 \mathrm{~cm} \times 28 \mathrm{~cm}$. The detector box was constructed to accept a number of different cameras, but we will primarily use one of three different Axiom cameras. Each of these cameras has a 9- $\mu \mathrm{m}$ detector element pitch. Two cameras have a 12-bit dynamic range and either $1536 \times 1024$ or $768 \times 512$ detector elements. We also can use a 16-bit $768 \times 512$ detectors camera. This provides an inherent spatial resolution and dynamic range gain over previously used 8-bit, $\sim 500 \times 500$ detector element cameras. A linear drive stage mounted under the turning mirrors allows precision positioning of the plane of focus within the glass scintillator. Improved spatial resolution imaging with high-energy $x$-rays has been demonstrated with the new imaging system. 


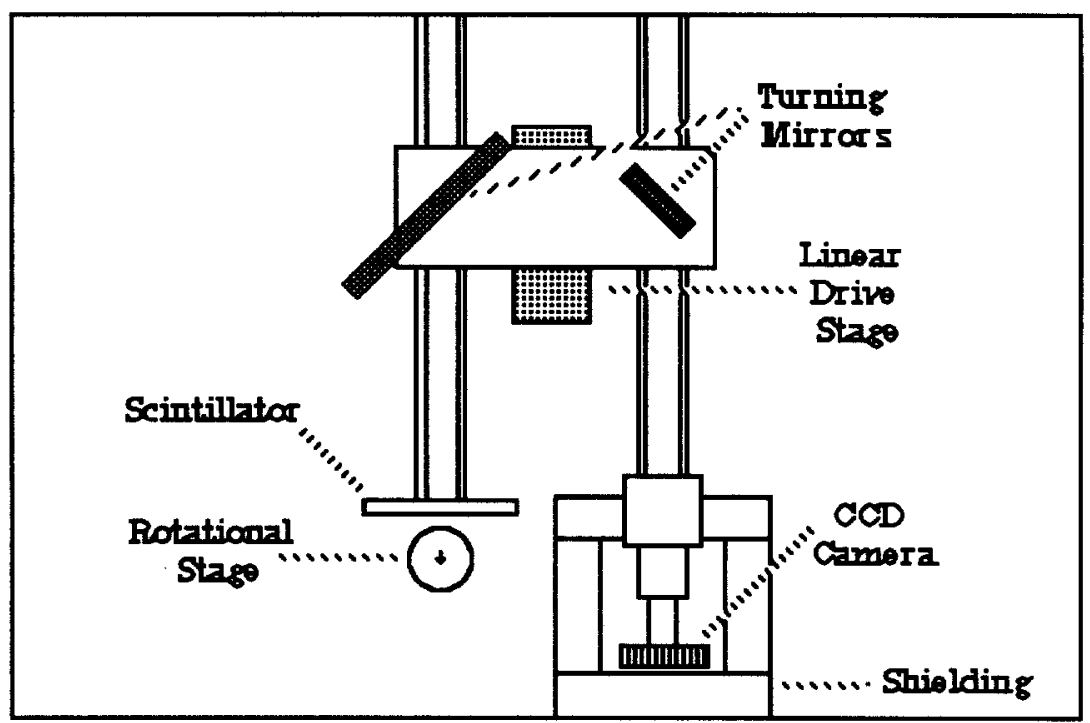

Figure 6. Plan view of the high-energy DR/CT system.

\section{Algorithm R\&D}

Our algorithm work has been concentrated on developing a single-pass limited-data CT reconstruction algorithm. We adapted a constrained conjugate gradient (CCG) CT algorithm [GOO94, GO093] to fit the ROI CT problem geometry, where only a small outer portion of the object is illuminated during each projection. This algorithm gives us the ability to apply upper and lower bounds to the solution, as well as the ability to fix variables to known values using priori information. For the purpose of using simulations to compare ROI CT with conventional full-field-of-view CT, we have assumed a camera with 100 detectors $^{\dagger}$. In the conventional CT case, the field-of-view of the detectors covers the entire object to be imaged. In the ROI CT case, the detectors cover only a small tangential region of the object. The original object consists of three concentric bands of material. The outer band has an attenuation value of 1 , the middle band a value of 4 , and the inner band a value of 2 . The interior of the object has an attenuation value of zero. Several small voids (attenuation 0 ) have been placed on the right side of the object.

The full-field-of-view convolution backprojection (CBP) reconstruction represents the typical resolution we could expect using conventional CT to perform the reconstruction. The resolution is poor: the transitions between the bands are blurry and the voids are blurry and spatially distorted. The interior of the CBP result is non-zero, as well. The ROI CCG reconstructed image has higher resolution than the CBP image, but suffers from high-frequency noise in the bands. Also, the voids are badly distorted. The noise and distortion are due to the ill-posed nature of the limited-data reconstruction. We are working to improve the result by introducing relaxation to the algorithm.

Other image reconstruction processes were investigated. In one investigation, an image was formed by reconstructing the limited-data projections after their average background value was subtracted. This forces the algorithm to concentrate on the deviations from the

${ }^{\dagger}$ The small number of detector elements, i.e., 100 , was used to decrease the computation time required for the simulation studies and we do not expect this to affect the study results when we scale up to 1000 and 1500 detector elements. 
background (the voids) as opposed to the background itself (the uniform bands). The spatial resolution is quite good and the circular shape of the voids is preserved. An image of the uniform bands is produced by reconstructing the average background projection at each angle. A final result is synthesized by subtracting the void image from the background image. The resolution of this difference image is good (although it too suffers from high frequency noise), the void shapes are preserved, but the value inside the voids is slightly off.

\section{Summary}

We have performed Monte Carlo calculations to study the interaction of $x$-rays with the detector components. These results reveal that low mass is best and were used to design the DR/CT detector. The scintillator modeling has been used to determine the scintillator characteristics and optimum focus and depth of field. The detector was fabricated at the end of this LDRD project. Improved spatial resolution imaging with high-energy $x$-rays has been demonstrated with the new imaging system. The detector system is currently being used in support of the Enhanced Surveillance Program (ESP) LL-ESP-96-29High Resolution X-ray Tomography project.

We have developed a single-pass algorithm based on a CCG technique. Preliminary results are encouraging. Capability to reconstruct only defects seems to perform best. It is not clear that a single pass technique will provide the desired results and how best to use prior information and how it effects the desired results.

In our future work, we will obtain scans of phantoms to validate the scanner before we perform studies of weapons components. For the algorithm studies we need to develop other simulations that include gaps and inclusions. We will concentrate on improving the single-pass algorithm, developing a two-pass algorithm, and comparing the two. Lastly, the scintillator and $x$-ray scatter may be the limiting factor for meeting the spatial resolution requirements.

\section{References}

[FRY96] Fry, David A. and Harold Berger, "Final Report Performance Characterization of Advanced Detectors for X-ray Imaging”, LACP96-33, 9 February, 1996.

[GO093] Goodman, D.M., E.M. Johansson, T.W. Lawrence, "On Applying the Conjugate Gradient Algorithm to Image Processing Problems", in Multivariate Analysis: Future Directions, C.R. Rao, Ed, Elsevier Science Publishers, Amsterdam, The Netherlands, 1993.

[GO094] Goodman, D.M., J. Kolman, S.G. Azevedo, "Image Reconstruction with Optimal Constraints", in Engineering Research, Development and Technology Thrust Area Report FY94, Lawrence Livermore National Laboratory, UCRL53868-94.

[PER96] Perkins, Dwight E., Presented at the WANTO Conference, Lawrence Livermore National Laboratory, Livermore, CA, May 1996. 
[TART95] Cullen, Dermott, E., Arthur L. Edwards, and Ernest F. Plechaty, "TART95: A coupled Neutron-Photon Monte Carlo Transport Code," UCRL-MA-121319, Lawrence Livermore National Laboratory, Livermore, CA, July 4, 1995.

[TER92] Glenn Tersell, private communication, Lawrence Livermore National Laboratory, Livermore, CA, 1992. 


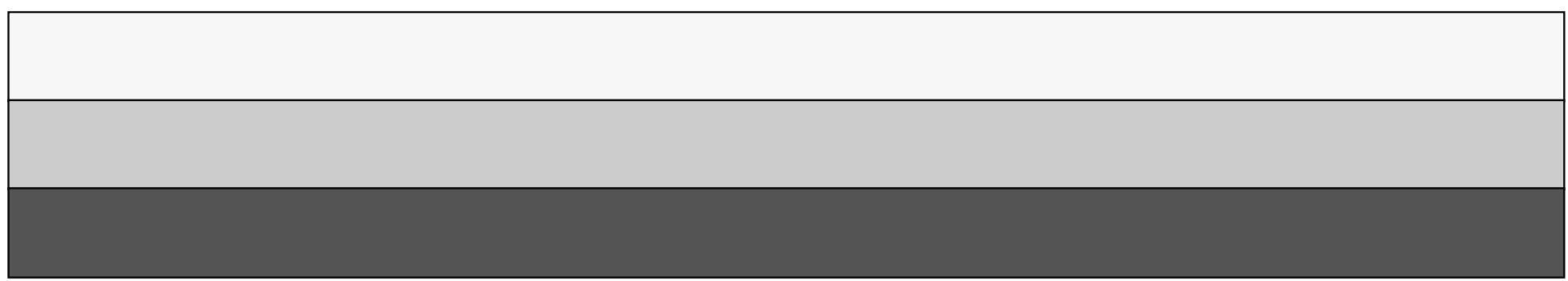

